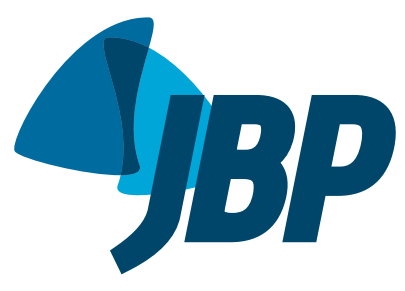

\title{
Educational interventions to improve inhaler techniques and their impact on asthma and COPD control: a pilot effectiveness- implementation trial
}

\author{
Tiago Maricoto ${ }^{1}$, Sofia Madanelo ${ }^{2}$, Luís Rodrigues ${ }^{3}$, Gilberto Teixeira ${ }^{3}$, \\ Carla Valente ${ }^{3}$, Lília Andrade ${ }^{3}$, Alcina Saraiva ${ }^{3}$
}

1. Unidade de Cuidados de Saúde Personalizados - UCSP - Aveiro I, Centro de Saúde de Aveiro, Aveiro, Portugal.

2. Unidade de Saúde Familiar de Santa Joana, Centro de Saúde de Aveiro, Aveiro, Portugal.

3. Departamento de Pneumologia, Centro Hospitalar do Baixo Vouga, Aveiro, Portugal.

Submitted: 25 March 2016.

Accepted: 21 July 2016.

Study carried out in the Departamento de Pneumologia, Centro Hospitalar do Baixo Vouga, Aveiro, Portugal.

\begin{abstract}
To assess the impact that educational interventions to improve inhaler techniques have on the clinical and functional control of asthma and COPD, we evaluated 44 participants before and after such an intervention. There was a significant decrease in the number of errors, and 20 patients (46\%) significantly improved their technique regarding prior exhalation and breath hold. In the asthma group, there were significant improvements in the mean $\mathrm{FEV}_{1}, \mathrm{FVC}$, and PEF (of $6.4 \%, 8.6 \%$, and $8.3 \%$ respectively). Those improvements were accompanied by improvements in Control of Allergic Rhinitis and Asthma Test scores but not in Asthma Control Test scores. In the COPD group, there were no significant variations. In asthma patients, educational interventions appear to improve inhaler technique, clinical control, and functional control.
\end{abstract}

Keywords: Asthma, Pulmonary disease, chronic obstructive; Nebulizers and vaporizers.
Inhalation therapy is the most effective modality for the treatment of asthma and COPD. The various types of inhalers available on the market have different specifications and require different techniques, leading to a number of errors in performance, thus reducing treatment efficacy and adherence. Up to $76 \%$ of patients make some type of error in their inhaler technique. ${ }^{(1)}$ Growing evidence suggests that educational interventions and reviews of the technique have a positive impact on disease control. $(2,3)$ This study was performed in the context of an interventional trial, with the aim of assessing the impact that an educational intervention to improve inhaler technique has on the clinical and functional control of asthma and COPD. Preliminary results containing the cross-sectional baseline analysis of the patients enrolled in the trial have previously been published. ${ }^{(4)}$ The results suggest a significant relationship between the number of errors committed and the level of clinical and functional control achieved in asthma patients.

We included patients with asthma or COPD, all of whom were receiving treatment with at least one inhaler device. The patients were evaluated in two different visits, with a six- to eight-month interval between visits. After the first visit, the patients were taught how to use their devices correctly. The inhaler technique was evaluated only for the main treatment device, and the use of a device for symptom relief was therefore not evaluated. The variables under study were demographic data; clinical control-by means of the Asthma Control Test (ACT), Control of Allergic Rhinitis and Asthma Test (CARAT), modified Medical Research Council (mMRC) scale, and COPD Assessment Test (CAT); functional control-absolute FEV1 (in percentage of the predicted value and in $\mathrm{mL}$ ), PEF (in percentage of the predicted value and in $\mathrm{mL}$ ), FVC (in $\mathrm{mL}$ and in percentage of the predicted value), $\mathrm{FEV}_{1} / \mathrm{FVC}$ ratio, $\mathrm{FEF}_{25-75 \% \text {, }}$ and $\mathrm{SpO}_{2}$; and number of steps of the inhaler technique correctly performed (per definition, step 1: device actuation; step 2: exhalation; step 3: inhalation; and step 4: a 5-10 s breath hold). The data were compiled in Microsoft Office Excel 2010, and statistical analyses were performed with IBM SPSS Statistics software package, version 20.0 (IBM Corporation; Armonk, NY, USA). The level of significance was set at $p<0.05$.

Of the initial 62 patients invited to participate in the study, 18 dropped out. Therefore, 44 patients were included in the follow-up re-evaluation (mean age, $59 \pm 16$ years). Of the 44 patients in the sample, 21 (47.8\%) were men, and 23 (52.2\%) were women. Among the 44 patients, 23 (52.2\%) and 21 (47.8\%) were diagnosed with asthma and COPD, respectively. In the first visit, a small number of technical errors was significantly associated with previous teaching of inhaler technique ( $p<0.05$; Fisher's exact test) and with age ( $p<0.05 ; R=0.13$; Pearson's correlation coefficient), although not with the number of years since diagnosis (Pearson's correlation coefficient). There was an association between a smaller number of errors and better clinical control in the asthma group, indicated by the ACT scores (maximum mean difference, 11.6 points) and CARAT scores (maximum mean difference, 12.3 points; $\mathrm{p}<0.05 ;$ ANOVA). However, although a similar graphic 
pattern was found in the COPD group, the differences within that group were not statistically significant.

Figure 1 includes the main results obtained. At the second visit, there was a significant decrease in the number of errors after the educational session, with a mean decrease of 0.7 errors (range, $0.29-1.02 ; \mathrm{p}<$ 0.05 ; t-test for paired samples). Among the 44 patients, $20(46 \%)$ improved their inhaler technique, with significant results found in step 2 (relative improvement of $45.5 \%$ ) and step 4 (relative improvement of $52 \%$; t-test for paired samples for both). In the asthma group, there were post-intervention improvements in the functional parameters (in percentages of the predicted values): the mean FEV $_{1}$ increased from $-10.26 \%$ to $-2.52 \%$ (a relative improvement of $6.4 \%$ ); the mean FVC increased from $-12.99 \%$ to $-4.14 \%$ (a relative improvement of $8.6 \%$ ); and the mean PEF increased from $-14.35 \%$ to $-2.35 \%$ (a relative improvement of $8.3 \%$ ). Comparing the preand post-intervention functional parameter values, we found the differences to be significant ( $p<0.05$ for all; t-test for paired samples). In addition, improved inhaler technique was significantly correlated with an improvement in the CARAT score $(O R=14.4 ; p$ $<0.05$; Fisher's exact test), although not in the ACT score. However, regarding the asthma group, if we defined a $>4$ point increase in the CARAT score as a clinically significant improvement in control, we would find that only 5 participants $(23.8 \%)$ met the criterion for such improvement. Using the same approach, if we established a $>3$ point increase in the ACT score as a clinically significant improvement in control, we would find that only 8 participants $(38.0 \%)$ met the criterion. In the COPD group, there were no significant variations in clinical or functional control variables. However, if we defined $a>1$ point increase in the mMRC score as a clinically significant improvement in control, we would find that 6 patients $(28.6 \%)$ met the criterion. If we defined $a>2$ point increase in the CAT score as a clinically significant improvement in control, we would find that 5 participants $(23.8 \%)$ met the criterion.

In a post-hoc analysis of the functional parameters, in absolute values $(\mathrm{mL})$, we found a statistically significant improvement in $\mathrm{FEV}_{1}$ in the COPD group (mean increase, $145.7 \mathrm{~mL}$; 95\% CI: 11.7-279.8; $\mathrm{p}=$ 0.035 ; t-test for paired samples). In the asthma group, there was improvement, although the difference was not statistically significant (mean increase, $105.9 \mathrm{~mL}$; 95\% CI: -47.6 to $259.4 ; p=0.17$; t-test for paired samples). For PEF (in $\mathrm{mL}$ ), there were improvements that were found to be statistically significant in the asthma group (mean increase, $460 \mathrm{~mL}$; 95\% CI: 30.9-890.0; $p=0.037$; t-test for paired samples) but not in the COPD group (mean increase, 212.9 $\mathrm{mL} ; 95 \% \mathrm{CI}$ : -184.0 to 609.7 ; $\mathrm{p}=0.276$; t-test for paired samples).

These results show that most patients make errors in the inhaler technique, and that seems to be associated with age and previous training regarding the technique.
Younger patients made fewer errors, which might be due to differences in literacy levels. The small sample size might have reduced the statistical power of some findings, such as the association with the number of years since diagnosis. Our results also show that proper inhaler technique was associated with better clinical control in asthma patients, and improving the technique could also affect functional parameters, mainly those directly related to airway obstruction, such as $\mathrm{FEV}_{1}$, FVC, and PEF. When comparing CARAT with ACT scores, we found that the former was more sensitive in detecting such changes, possibly because CARAT is a more comprehensive clinical test. ${ }^{(5)}$ In patients with COPD, no significant improvements were observed, either in clinical control or in functional parameters (in percentage of the predicted value), which, as previously mentioned, might be due to the small sample size, or even to the pathophysiological differences between the diseases. However, some of those patients showed clinically significant improvement after the educational intervention, which supports the hypothesis that larger samples and continuous training might be more accurate in detecting statistically significant differences. However, we found some improvement in functional performance ( $F E V_{1}$ in $\mathrm{mL}$ ). Although that finding was statistically significant, $\mathrm{FEV}_{1}$ in percentage of the predicted value was not. That discrepancy can be explained by the fact that over $50 \%$ of our COPD patients were classified as having stage 1 or 2 disease according to the Global Initiative for Chronic Obstructive Lung Disease criteria. Therefore, those patients had higher $\mathrm{FEV}_{1}$ values, which meant that a relative increase of $145.7 \mathrm{~mL}$ represented a small proportion of the total value, leading to the underestimation of this finding. In addition, patients with severe COPD are older than are those with milder forms of the disease and are therefore less sensitive to educational interventions to improve inhaler technique and, consequently, to their potential beneficial impact. In our study, although adjusting for age had no effect on the results of the statistical analysis of functional and clinical impacts, age was found to be a determinant of the performance on inhaler technique tests. Further studies should be performed in elderly populations, because they have particular characteristics that might influence the inhaler technique and its impact. The fact that airway obstruction is more reversible in asthma patients might also justify a greater difference in their functional measurements, as well as in their perception of respiratory symptoms. These findings are consistent with those obtained in another study. ${ }^{(6)}$ A large trial is currently ongoing in order to address the impact of different educational approaches on patients with COPD, and its results are expected to be enlightening. ${ }^{(7)}$

The establishment of a query surveillance program in the primary health care setting could represent one solution to the problem of limited patient knowledge of proper inhaler technique, allowing the treatment of a greater number of patients in the general population. In such a network, other health care professionals, such as physicians from other specialties, respiratory 
(A)

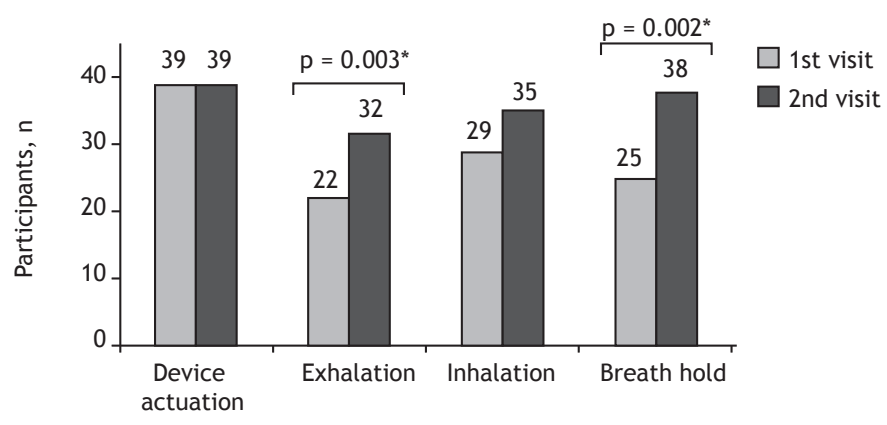

(B)

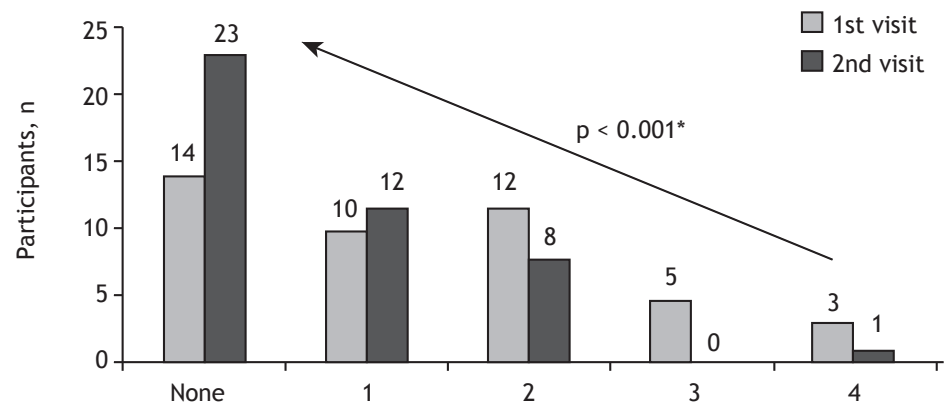

Errors, $n$

(C)

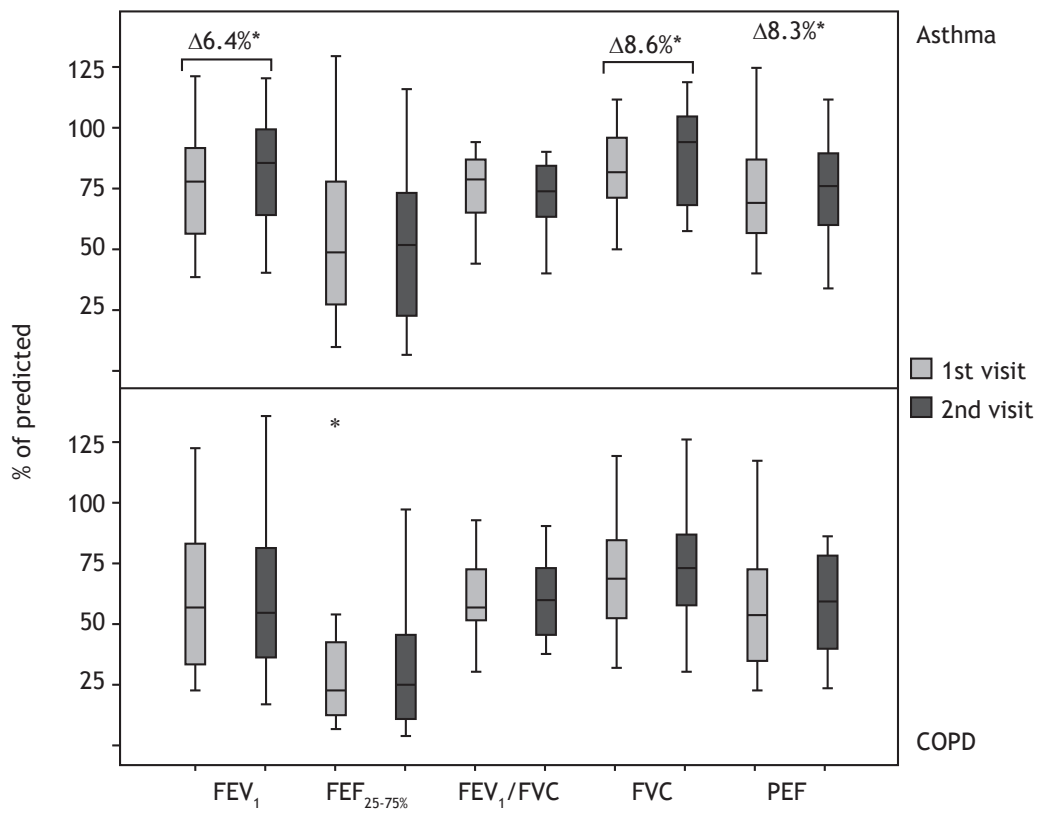

Figure 1. Number of participants using the inhaler device correctly, according to the pre-established steps (in A); the number of errors committed in the inhaler technique at the first and second visits (in $\mathrm{B}$ ); and the functional control parameters at the first and second visits in the asthma and COPD groups (in C). ${ }^{*} p<0.05$; t-test for paired samples.

therapists, nurses, and pharmacists, could be involved in the education of those patients. Some studies have tested the teaching of inhaler techniques and suggested that rechecking and regular refresher courses are needed, because proper inhalation technique deteriorates after the initial intervention. ${ }^{(8,9)}$ The loss of skills is accompanied by the deterioration of asthma control as soon as three months after the intervention. Although various strategies can be used in order to provide this type of educational intervention, it is best delivered through verbal instructions and physical demonstration of the technique by a skilled educator, 
either on video or face to face, as we did in our study. ${ }^{(8,9)}$ Some other educational techniques should be studied and tested in studies with appropriate designs, such as those involving large cohorts that provide large follow-up data sets. In addition, alternative ways of integrating multidisciplinary teams and different health care professionals should be addressed, as should the effect of training those professionals to properly educate their patients in the use of their inhalers.

We conclude that educational interventions to teach inhaler techniques improve the performance of patients, leading to improvements in the clinical and functional control of asthma. In COPD, the pathophysiological hallmark of irreversible obstruction might limit this benefit. Further studies on asthma should focus on patient outcomes that matter, in terms of the impact that educational interventions to teach inhaler techniques have on patient performance of those techniques, such as decreasing the risk of exacerbations. The same approach should be adopted for COPD patients, adjusting sample sizes to optimize the statistical power of randomized control trials and obtain good evidence.

\section{REFERENCES}

1. Molimard M, Raherison C, Lignot S, Depont F, Abouelfath A, Moore N. Assessment of handling of inhaler devices in real life: an observational study in 3811 patients in primary care. J Aerosol Med. 2003;16(3):249-54. https://doi.org/10.1089/089426803769017613

2. Lavorini F, Magnan A, Dubus JC, Voshaar T, Corbetta L, Broeders M, et al. Effect of incorrect use of dry powder inhalers on management of patients with asthma and COPD. Respir Med. 2008:102(4):593604. https://doi.org/10.1016/j.rmed.2007.11.003

3. Giraud V, Allaert FA, Roche N. Inhaler technique and asthma: feasability and acceptability of training by pharmacists. Respir Med. 2011;105(12):1815-22. https://doi.org/10.1016/j.rmed.2011.07.004

4. Maricoto T, Rodrigues LV, Teixeira G, Valente C, Andrade L, Saraiva A. Assessment of Inhalation Technique in Clinical and Functional Control of Asthma and Chronic Obstructive Pulmonary Disease. Acta Med Port. 2015;28(6):702-7. https://doi.org/10.20344/amp.5905

5. Domingues M, Amaral R, Fonseca JA, Azevedo P, Correia-deSousa J. Assessment of asthma control using CARAT in patients with and without Allergic Rhinitis: A pilot study in primary care. Rev Port Pneumol (2006). 2016;22(3):163-6. https://doi.org/10.1016/j. rppnen.2015.10.014

6. Press VG, Arora VM, Shah LM, Lewis SL, Charbeneau J, Naureckas $E T$, et al. Teaching the use of respiratory inhalers to hospitalized patients with asthma or COPD: a randomized trial. J Gen Intern Med. 2012;27(10):1317-25. https://doi.org/10.1007/s11606-012-2090-9

7. Leiva-Fernández F, Leiva-Fernández J, Zubeldia-Santoyo F, GarcíaRuiz A, Prados-Torres D, Barnestein-Fonseca P. Efficacy of two educational interventions about inhalation techniques in patients with chronic obstructive pulmonary disease (COPD). TECEPOC: study protocol for a partially randomized controlled trial (preference trial). Trials. 2012;13:64. https://doi.org/10.1186/1745-6215-13-64

8. Basheti IA, Armour CL, Bosnic-Anticevich SZ, Reddel HK. Evaluation of a novel educational strategy, including inhaler-based reminder labels, to improve asthma inhaler technique. Patient Educ Couns. 2008;72(1):26-33. https://doi.org/10.1016/j.pec.2008.01.014

9. Crompton GK, Barnes PJ, Broeders M, Corrigan C, Corbetta L, Dekhuijzen $R$, et al. The need to improve inhalation technique in Europe: a report from the Aerosol Drug Management Improvement Team. Respir Med. 2006;100(9):1479-94. https://doi.org/10.1016/j. rmed.2006.01.008 